\title{
Tuberculosis como causa de adenopatías cervicales
}

\author{
Tuberculosis as a cervical lymphadenopathy cause
}

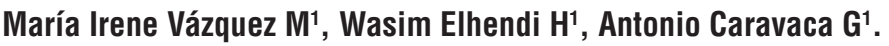

\begin{abstract}
RESUMEN
La tuberculosis (TBC) es una enfermedad infecto-contagiosa de distribución mundial causada por Mycobacterium tuberculosis, y otras micobacterias atípicas. La afectación ganglionar es tardía y sus manifestaciones clínicas asociadas suelen ser inespecíficas, por eso, el diagnóstico de tuberculosis ganglionar a menudo se retrasa y es un hallazgo inesperado en numerosas ocasiones. Este artículo pretende realizar una revisión bibliográfica sobre la tuberculosis ganglionar y hacer hincapié en que la TBC ha de ser tenida en cuenta como diagnóstico diferencial en las masas cervicales, que muchas veces se presentan con escasa sintomatología acompañante. En este artículo presentamos dos casos de TBC ganglionar diagnosticados en nuestro servicio en los últimos meses, ambos casos se manifestaron exclusivamente como masa cervical de crecimiento lento, sin síntomas pulmonares acompañante y fueron diagnosticados de TBC tras el estudio anatomopatológico resultante de la exéresis quirúrgica de la lesión.
\end{abstract}

Palabras clave: Tuberculosis, ganglio linfático.

\begin{abstract}
The tuberculosis (TB) is an infect-contagious worldwide distribution disease caused by Mycobacterium Tuberculosis and other atypical Mycobacteria. Lymph node involvement is late, and its associated clinical manifestations are usually unspecifics, therefore the diagnosis of tuberculosis lymph node is often delayed and is an unexpected finding in numerous occasions. This article aims to carry out a literature review of lymph node tuberculosis and to emphasize that TB must be taken into account as differential diagnosis in cervical masses, which often occur with few associated symptoms. In this article we present two cases of lymph node TB diagnosed in our department in last months, both cases presented exclusively as cervical mass of slow growth, without any accompanying pulmonary symptoms and were diagnosed as TB after the surgical removal of the lesion and its histopathological study.
\end{abstract}

Key words: Tuberculosis, lymph node.

\footnotetext{
${ }^{1}$ Médico Otorrinolaringología, Hospital Punta de Europa. Algeciras, Cádiz España.
}

Recibido el 19 de noviembre, 2016. Aceptado el 29 de enero, 2017. 


\section{INTRODUCCION}

La tuberculosis es una de las enfermedades infecto-contagiosas de distribución mundial con tasas de mortalidad más altas, acompañada de otras patologías como infecciones respiratorias agudas, VIH y gastroenteritis ${ }^{1}$. En los últimos años se ha producido un incremento de las infecciones tuberculosas, asociado sobre todo a situaciones sociales como la inmigración y núcleos poblacionales desfavorecidos, pacientes inmunodeprimidos y sobre todo asociado al VIH, grupo en el que la TBC es 500 veces más frecuente que en la población sana ${ }^{2}$. Entre los órganos extrapulmonares más afectados por la tuberculosis en pacientes VIH están los ganglios linfáticos, tanto periféricos como intratorácicos 0 intraabdominales. La presencia de adenopatías significativas en cualquier territorio junto con fiebre $\mathrm{u}$ otras manifestaciones en pacientes positivos al VIH debe sugerir como primera posibilidad diagnóstica la TBC ${ }^{3}$.

El bacilo Mycobacterium tuberculosis es el responsable del $10 \%-20 \%$ de los casos de linfadenitis cervical micobacteriana ${ }^{4}$. La presentación más frecuente de tuberculosis en cabeza y cuello es la linfadenitis cervical, que constituye el $95 \%$ de los casos ${ }^{5}$. La afectación ganglionar aparece lentamente, unos 4-6 meses tras la primoinfección, en forma de adenopatías multilobuladas, unilaterales e indoloras, de crecimiento lento, lo que dificulta inicialmente el diagnóstico de tuberculosis, al tener que realizar diagnóstico diferencial con múltiples patologías tanto infecciosas como tumorales originarias de masas laterocervicales.

Nuestro hospital se encuentra situado en el Campo de Gibraltar. Algeciras es una ciudad multicultural, paso para numerosas personas que viajan desde Europa a África, especialmente a zonas del norte de África y viceversa, por lo que estamos en contacto con un continuo flujo de personas de prácticamente todo el mundo. En el caso de la TBC, la inmigración, zonas desfavorecidas, así como la infección VIH y otros estados de inmunosupresión, se consideran factores de riesgo para la misma. Nosotros presentamos en este artículo dos casos de pacientes, que solicitando atención médica en nuestro hospital, son diagnosticados tras cervicotomía de tuberculosis ganglionar cervical.

\section{CASOS CLINICOS}

\section{Caso número 1}

Paciente de 10 años de edad, sin antecedentes de interés, que acude a nuestro servicio por presentar aumento de volumen laterocervical izquierdo, de 6 meses de evolución aproximadamente, con buena respuesta inicial al tratamiento antibiótico empírico en su país de origen, pero con persistencia posterior. No refiere síntomas asociados de astenia, febrícula, tos o sudoración nocturna. No refiere antecedentes de interés, vacunada correctamente. No refiere antecedentes de contacto con tosedores habituales ni personas enfermas.

Se solicita una TAC por parte del servicio de otorrinolaringología que informa de "extensa lesión laterocervical izquierda $(10 \mathrm{~cm}$ de diámetro craneocaudal $\times 30 \mathrm{~mm}$ anteroposterior $\times 25 \mathrm{~mm}$ transverso) predominantemente quística, multiloculada, con polos sólidos, que contacta en su zona superior con el lóbulo parotídeo profundo, extendiéndose caudalmente, hasta el borde posterior del tiroides. Se sitúa medial al músculo ECM que parece no estar infiltrado y desplaza medialmente al paquete vascular yúgulo-carotideo con importante compresión de la vena yugular interna, que queda casi obliterada en su tercio superior". Sugieren radiológicamente como posibles diagnósticos diferenciales el linfangioma quístico, quiste branquial complicado 0 extenso conglomerado adenopático necrosado (Figura 1).

La paciente es intervenida bajo anestesia general, realizándose cervicotomía vía RAHI (incisión retroauricular siguiendo la línea del cabello). Durante la intervención quirúrgica nos encontramos por debajo de ECM y en íntima relación con el paquete vascular cervical, llegando hasta la zona subdigástrica, un complejo poliadenopático, con adenopatías friables, que se rompen con facilidad dejando ver un contenido caseoso (Figura 2). Tras la exéresis de varias adenopatías, se remiten para estudio anatomopatológico intraoperatorio y microbiológico por sospecha de TBC ganglionar.

El resultado anatomopatológico (biopsia intraoperatoria) informa de linfadenitis granulomatosa necrotizante sugestiva de tuberculosis. El resultado anatomopatológico definitivo de la pieza quirúrgica confirma dicho resultado. 


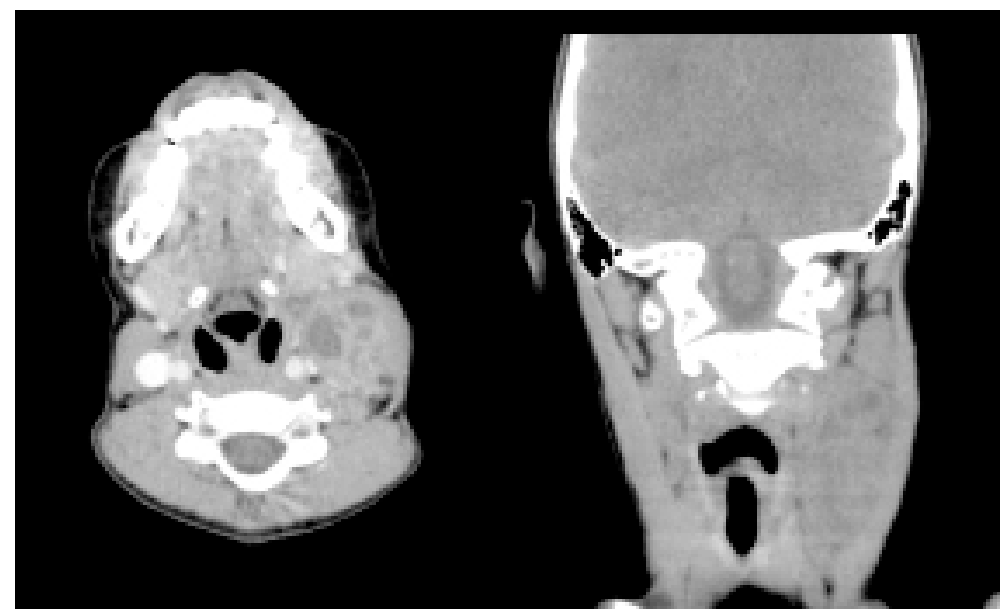

Figura 1. TAC axial y coronal. Se observa extensa lesión sólido-quística polilobulada.

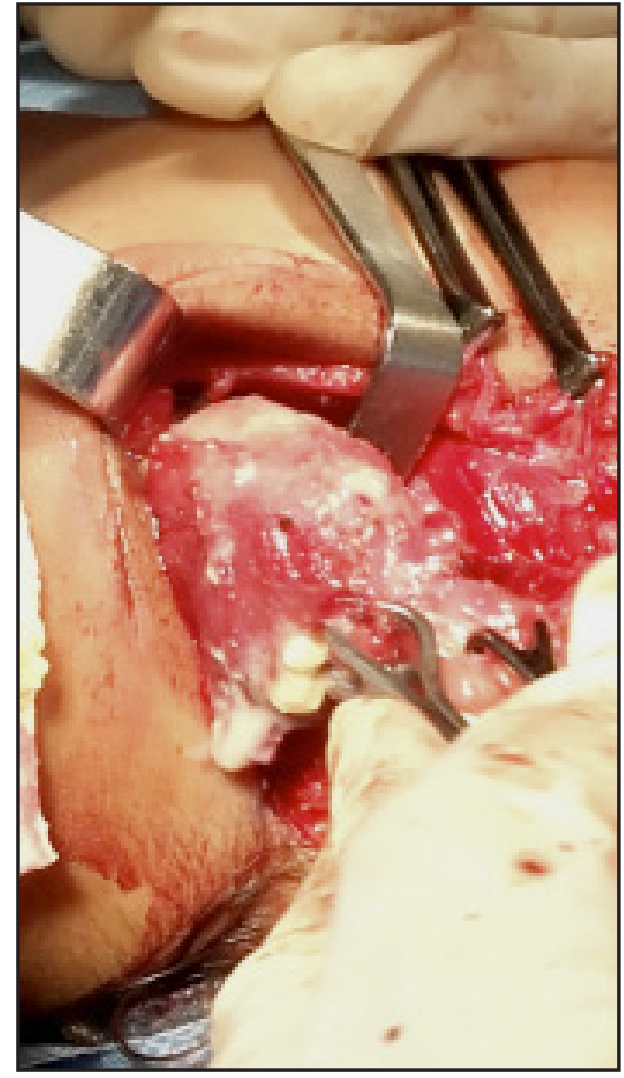

Figura 2. Imagen intraoperatoria. Tumoración gran tamaño, abscesificada y caseosa.
Tras realizar pruebas complementarias por parte del servicio de pediatría, se confirma la presencia de BAAR (Bacilo ácido-alcohol resistente) y con el diagnóstico de tuberculosis ganglionar se inicia tratamiento tuberculostático con isoniacida, rifampicina, piracinamida y etambutol con buena evolución de la paciente y desaparición de las adenopatías laterocervicales.

\section{Caso número 2}

Paciente de 65 años, que comienza desde hace 2 meses con cuadro de aumento de volumen laterocervical izquierdo. Refiere clínica previa asociada de tos y fiebre que fue catalogado en su país de origen como infección respiratoria y tratada empíricamente como tal con mejoría. Como antecedentes personales la paciente refiere HTA y dislipemia con buen control.

En la exploración otorrinolaringológica, incluida la rinofibrolaringoscopía, no se evidencia lesión primaria que justifique el proceso. Se realizan pruebas de imagen con TAC cervical que confirma la existencia de una adenopatía voluminosa localizada en el espacio II submandibular izquierdo de $33 \times 20 \times 19 \mathrm{~mm}$ captante de contraste con múltiples focos internos de tipo quístico o necrótico asociados a microcalcificaciones. Existen también múltiples adenopatías dependientes de todas las 
cadenas cervicales en general subcentrimétricas destacando una a nivel III/IV izquierdo de similares características a la del nivel II y unas medidas de $28 \times 15 \times 12$. Se recomienda valorar proceso granulomatoso sin poder descartar proceso neoplásico (Figura 3).

Se realiza cervicotomía izquierda, con exéresis de una adenopatía bien delimitada, encapsulada, situada a nivel IIb/III laterocervical izquierda por debajo del ECM y en contacto con el paquete vascular del cuello.

El resultado anatomopatológico informa de linfadenitis granulomatosa necrotizante sugestiva de tuberculosis.

La paciente es enviada al servicio de enfermedades infecciosas donde completan su estudio e inician tratamiento tuberculostático con Rifinah $^{\circledR}$ (isoniacida + rifampicina) con buena evolución.

\section{DISCUSION}

La tuberculosis es una enfermedad infecciosa y contagiosa sistémica de distribución mundial causada por Mycobacterium tuberculosis y por otras micobacterias atípicas. Se estima que un tercio de la población mundial está infectado por Mycobacterium tuberculosis, del cual 5\%-10\% padece la enfermedad en algún momento ${ }^{5}$. El ser humano es el único vector que actúa como reservorio y como agente transmisivo.
Normalmente la enfermedad se localiza en los pulmones, pero puede afectar prácticamente a cualquier órgano del cuerpo humano; se denomina entonces TBC extrapulmonar. Una de las formas más frecuentes de presentación de la TBC extrapulmonar es la de los ganglios linfáticos, cuya incidencia es especialmente elevada en los pacientes con infección por VIH. La enfermedad extrapulmonar puede afectar a ganglios linfáticos (incluida la adenopatía hiliar), médula ósea, hígado, riñón, meninges, pericardio y tracto gastrointestinal. Otras afecciones más raras son osteoarticular, peritonitis, laringitis, etc... ${ }^{6}$. Actualmente la tuberculosis extrapulmonar constituye el $25 \%$ del total de la patología tuberculosa, de la cual, la afectación ganglionar cervical constituye el 35\%-50\%². La afectación ganglionar aparece tardíamente, unos 4-6 meses tras la primoinfeccion, en forma de adenopatías multilobuladas, unilaterales, indoloras y de crecimiento lento. Las adenopatías tienden a crecer gradualmente y en principio son de consistencia rígida e indolora. Con el tiempo pueden sufrir necrosis, fluctuar y presentar signos inflamatorios con fistulización y drenaje de caseum al exterior, lo que se conoce como escrófula ${ }^{3}$.

El cuadro clínico de afectación de tuberculosis en el área de cabeza y cuello suele ser confuso, y hay que realizar diagnóstico diferencial con otras enfermedades granulomatosas crónicas 0 con neoplasias, sobre todo en caso de procesos inflamatorios con mala respuesta al tratamiento 0

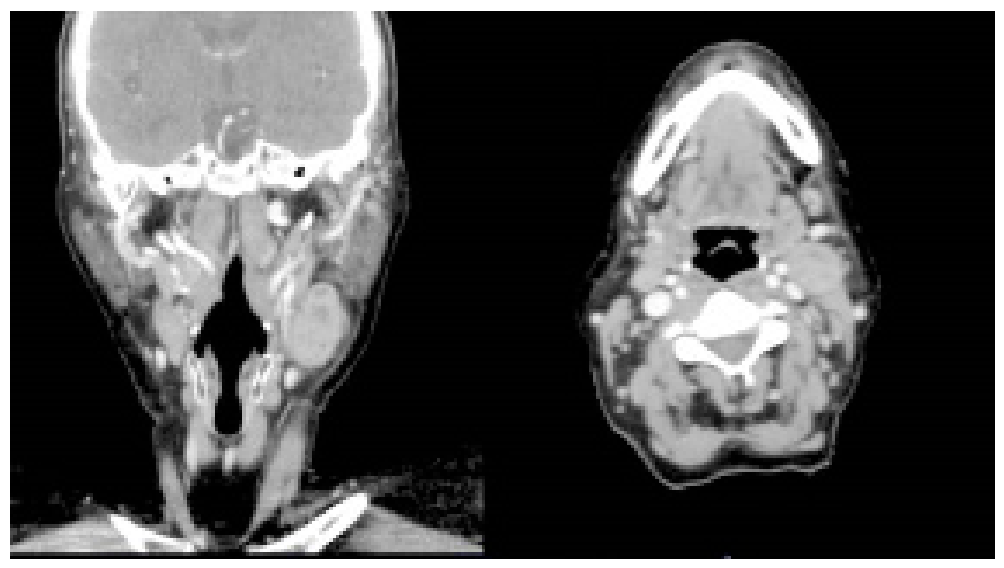

Figura 3. TAC axial y coronal de cuello. Imagen de adenopatía encapsulada. 
si hay asociadas adenopatías, úlceras o fístulas. Es importante recordar que el diagnóstico de un caso de tuberculosis infantil es indicativo de mala estrategia para el diagnóstico y tratamiento de adultos bacilíferos, que viven en una comunidad aún sin diagnosticarse ${ }^{7}$.

La biopsia- exéresis es la actitud clásica, que permite realizar un diagnóstico en el $100 \%$ de los casos y aislar el microorganismo en cultivo en el 60\%-90\% de los casos, siendo la PAAF (punciónaspiración con aguja fina) más controvertida ya que permite el diagnóstico histológico en $80 \%$ de los casos $^{8}$. Las formas extrapulmonares de tuberculosis tienen menos carga bacilar, sin embargo la PAAF debe realizarse en todos los casos de sospecha para determinar si la infección es por Mycobacterium tuberculosis o por micobacterias atípicas.

El tratamiento es médico con una duración de 6-9 meses, con antibióticos antituberculosos bactericidas (isoniacida, rifampicina) asociados a etambutol o pirazinamida durante los dos primeros meses $^{8,4}$. Este tratamiento tiene como objetivo la curación del paciente y la prevención de la transmisión ${ }^{9}$. La cirugía se reserva a aquellos casos de grandes conglomerados adenopáticos 0 abscesos fríos, o bien cuando es precisa una biopsia (siempre escisional y nunca realizando linfadenectomías amplias) $)^{2}$.

En los dos casos presentados en este artículo, el diagnóstico fue realizado tras la exéresis quirúrgica de la lesión. En el primer caso, se realiza una resección de varias adenopatías del gran conglomerado adenopático que la paciente presentaba. Con la elevada sospecha de TBC por el aspecto macroscópico intraquirúrgico que las adenopatías presentaban, se envía una biopsia intraoperatoria, confirmándonos nuestra alta sospecha de TBC cervical. En el segundo caso se realiza exéresis de la adenopatía más destacada y voluminosa observada en los estudios de imagen.

En ambos casos se realizó el diagnóstico definitivo de tuberculosis tras el estudio anatomopatológico de las adenopatías, puesto que inicialmente el diagnóstico diferencial planteado era amplio, ya que la clínica asociada era escasa. Se confirma lo ya expuesto en este artículo sobre la escasez de manifestaciones clínicas asociadas en la TBC ganglionar quedando patente la dificultad de los profesionales para realizar un diagnóstico precoz de la misma.

Teniendo en cuenta la escasa sintomatología de las formas ganglionares cervicales de la TBC, creemos que, en nuestra área geográfica al menos, todo otorrinolaringólogo pero también todo médico de Atención Primaria que atienda tanto a adultos como a niños con un proceso adenopático cervical, debe tener presente a la TBC como parte del diagnóstico diferencial, especialmente si han existido tratamientos empíricos previos que no han resuelto el problema.

\section{BIBLIOGRAFIA}

1. Rivera Cuello Mercedes. Enfermedades emergentes. BOL SPAO 2011; 51.

2. Rodríguez Pérez MA, Aguirre García F. Tuberculosis ganglionar cervical. ¿Pensamos en ella o nos sorprende? Rev Otorrinolaringol Cir Cabeza y Cuello 2012; 72: 69-74.

3. Juliá González-Martin J et al. Documento de consenso sobre diagnóstico, tratamiento y prevención de la tuberculosis. Arch Bronconeumol 2010; 46: 255-74.

4. Roda Aceituno M, Carmona Marietto A, Rodríguez

Briceño M. Infecciones cervicales. En: Navarro Paule MP, Pérez Aguilera R, Sprekelsen Gasso C. Manual de Otorrinolaringologia infantil. Elservier España. Barcelona 2012; pp 426-34.

5. Prado Caballero H, Rodríguez Valero M, Marmissolle DaguerRe G. Utilidad de las pruebas diagnósticas en tuberculosis del área otorrinolaringológica y de cabeza y cuello. An ORL MEXVOL.58, NUM. 2, 2013.

6. Campillos Páez M.T. et al. Tuberculosis ganglionar. Medicina General 2001; 35: 529-32.

7. Cruz I, Salcedo M. Tuberculosis ganglionar. Experiencia en el Instituto Nacional de 
Enfermedades Respiratorias. Arch Pediatr Urug 2011; 82(1): 18-22.

8. Alañón Fernández Ma, Crespo del Hierro J, Ruiz González M, Martínez Fernández A. Patología infecciosa e inflamatoria a nivel cervical. En:
Alañon Fernández MA et al. Tumoraciones cervicales. GlaxoSmithKline SA. Madrid: pp 69-84.

8. Peralta Fernández G. Tuberculois de cabeza y cuello. Acta Otorrinolaringol Esp 2009; 60(1): 59-66. 